\title{
High gastrointestinal permeability and local metabolism of naringenin: influence of antibiotic treatment on absorption and metabolism
}

\author{
Naiara Orrego-Lagarón ${ }^{1}$, Miriam Martínez-Huélamo ${ }^{2,3}$, Anna Vallverdú-Queralt ${ }^{2,3}$, \\ Rosa M. Lamuela-Raventos ${ }^{2,3}$ and Elvira Escribano-Ferrer ${ }^{1,3 *}$ \\ ${ }^{1}$ Department of Pharmacy and Pharmaceutical Technology, Faculty of Pharmacy, University of Barcelona, \\ Avgda Joan XXIII s/n, E-O8028 Barcelona, Spain \\ ${ }^{2}$ Nutrition and Food Science Department, XaRTA, INSA, Faculty of Pharmacy, University of Barcelona, E-O8028 Barcelona, \\ Spain \\ ${ }^{3}$ CIBER Fisiopatologia de la Obesidad y Nutrición (CIBEROBN), Instituto de Salud Carlos III, E-28029 Madrid, Spain \\ (Submitted 23 May 2014 - Final revision received 9 April 2015 - Accepted 14 April 2015 - First published online 17 June 2015)
}

\section{Abstract}

The present study aims to determine the permeability of naringenin in the stomach, small intestine and colon, to evaluate intestinal and hepatic first-pass metabolism, and to study the influence of the microbiota on the absorption and disposition of naringenin $(3.5 \mu \mathrm{g} / \mathrm{ml}$ ). A single-pass intestinal perfusion model in mice ( $n$ 4-6) was used. Perfusate (every $10 \mathrm{~min}$ ), blood (at $60 \mathrm{~min})$ and bile samples were taken and analysed to evaluate the presence of naringenin and its metabolites by an HPLC-MS/MS method. To study the influence of the microbiota on the bioavailability of naringenin, a group of animals received the antibiotic rifaximin ( $50 \mathrm{mg} / \mathrm{kg}$ per $\mathrm{d}$ ) for $5 \mathrm{~d}$, and naringenin permeability was determined in the colon. Naringenin was absorbed well throughout the gastrointestinal tract but mainly in the small intestine and colon (mean permeability coefficient $7.80(\mathrm{sD} 1.54) \times 10^{-4} \mathrm{~cm} / \mathrm{s}$ and $5.49(\mathrm{sD} 1.86) \times 10^{-4} \mathrm{~cm} / \mathrm{s}$, respectively), at a level similar to the highly permeable compound, naproxen $\left(6.39(\mathrm{sD} 1.23) \times 10^{-4} \mathrm{~cm} / \mathrm{s}\right)$. According to the high amounts of metabolites found in the perfusate compared to the bile and plasma, naringenin underwent extensive intestinal first-pass metabolism, and the main metabolites excreted were sulfates ( 84.00 (SD 12.14)\%), followed by glucuronides ( $8 \cdot 40$ (sD 5.67)\%). Phase II metabolites were found in all perfusates from $5 \mathrm{~min}$ of sampling. Mice treated with rifaximin showed a decrease in naringenin permeability and in the amounts of 4-hydroxyhippuric acid and hippuric acid in the lumen. Naringenin was well absorbed throughout the gastrointestinal tract and its poor bioavailability was due mainly to high intestinal metabolism.

Key words: Naringenin: Metabolism: In situ single-pass perfusion: Gastrointestinal tract: Rifaximin

Naringenin is the predominant flavanone found in citrus fruit and tomato, and an important compound in the human diet. Like other flavanones, it shows antioxidant, anti-inflammatory and anticarcinogenic activity, and the capacity to diminish blood lipids and cholesterol after oral administration ${ }^{(1)}$. An inhibitory effect of naringenin on the hepatitis $\mathrm{C}$ virus has also been reported ${ }^{(2)}$. A growing awareness of these properties, together with its great abundance in our diet, has stimulated interest in naringenin. The benefits for human health attributed to polyphenols in general and to naringenin in particular depend on their oral bioavailability ${ }^{(3,4)}$. However, the oral bioavailability of naringenin is very low, being less than $10 \%{ }^{(1)}$. One of the major factors influencing bioavailability is the absorption/permeability of the compound through the gastrointestinal tract. If ingested in a solid state, its solubility in physiological fluids could be a limiting factor for the rate and extent of absorption ${ }^{(5)}$. Moreover, two products with the same intestinal permeability could present differences in absorption due to variable dissolution in vivo. In this respect, ethanol at high concentration $(>30 \% \mathrm{v} / \mathrm{v})$ was shown to enhance the absorption of quercetin in rats ${ }^{(6)}$. Consequently, bioavailability essentially depends not only on the permeability of the compound through the gastrointestinal tract, but also on the elimination process by excretion and/or intestinal and hepatic metabolism, as well as the effect of microbial enzymes on these processes. An in-depth study of these processes could help to determine whether the benefits of naringenin for human health can be attributed to bioavailable naringenin or to its derived metabolites.

Abbreviations: NAR-GLU, naringenin glucuronide; NAR-SULF, naringenin sulfate; Peff, effective permeability coefficient.

*Corresponding author: E. Escribano-Ferrer, +349340 24578, email eescribano@ub.edu 
Flavonoids participate in both enteric and enterohepatic recycling: the former is enabled by enteric excretion of phase II conjugates, whereas the latter involves the hepatic excretion of the conjugates into the bile. Both recycling processes require efflux transporters such as multidrug resistance-associated protein 2 (MRP-2) $^{(1)}$ and bacterial enzymes that can hydrolyse the conjugates to regenerate the aglycons, which are then reabsorbed and recycled into the systemic circulation. In this context, the study of the influence of the microbiota on the disposition of naringenin is of great interest. Chabane et $a l^{(4)}$ elucidated the intestinal transport mechanism of naringenin and quercetin by means of an in vitro study (Caco-2 cells), but there is little information on the effect of intestinal metabolism on naringenin bioavailability. An attempt to shed light on this was made by Xu et $a l^{(1)}$, whose study in rats was centred on the glucoronidation pathway, providing no information about sulfates. Moreover, according to Jeong et ll $^{(7)}$, mice are more suitable animals for studying the disposition of naringenin, since the amounts of flavonoid sulfates (especially at lower concentrations) excreted by rats are insignificant, while in mice they are of the same order as in human plasma and urine. Thus, the mouse intestinal perfusion model for compounds excreted as sulfates and glucuronides, including flavonoids, has significant advantages over the rat model. Moreover, Escribano et al. ${ }^{(8)}$ found a good correlation ( $R$ 0.9631) between the mouse permeability coefficient of five model drugs in mice and the fraction of oral dose absorbed in human subjects, showing the capacity of this model to predict human absorption.

Based on these considerations, and given that naringenin is a dietary compound that can be ingested from different sources and/or matrices (e.g. juice, tomato sauce and high-fat diet), allowing absorption to start in the stomach and continue throughout the intestinal tract, the aims of the present study were: (1) to determine the solubility of naringenin under physiological $\mathrm{pH}$ conditions and its effective permeability coefficient (Peff) through the gastrointestinal tract, using an in situ single-pass perfusion technique in mice. As a model drug, and according to the Biopharmaceutics Classification System (BCS $)^{(5)}$, naproxen was assayed as a high-permeability reference compound; (2) to evaluate the intestinal and hepatic first-pass effect through the detection and quantification of metabolites in the lumen, bile and plasma; (3) to study the influence of antibiotic treatment on the colonic permeability of naringenin and intestinal metabolism.

\section{Materials and methods}

\section{Reagents and materials}

Naringenin, naproxen, phloroglucinol, 3-phenylpropionic acid, (4-hydroxyphenyl) acetic acid, hippuric acid, quinic acid, rifaximin, phenol red, casein from bovine milk, L-cystine hydrochloride monohydrate, D,L-methionine, choline chloride and dextrin from maize starch were provided by Sigma-Aldrich; naringenin-4-O-glucuronide (NAR-GLU) and naringenin-7-O-glucuronide (NAR-GLU) were from Cayman Chemical Company; 3-(4-hydroxyphenyl) propionic acid was from Extrasynthese; Hegsted salt mixture and Vitamin Diet Fortification Mixture were purchased from Group Taper; span 20 (Sorbitan monolaurate) and sodium carboxymethylcellulose from Fagron; and refined maize oil, saccharine and saccharose were purchased from a supermarket. Reagents, $\mathrm{ZnCl}_{2}, \mathrm{NaCl}$, sodium monohydrogen phosphate dehydrate, anhydrous disodium hydrogen phosphate, orthophosphoric acid and $\mathrm{NaOH}$ for analysis were provided by Panreac Química; and ethanol, methanol, acetonitrile and formic acid were purchased from Scharlau Chemie, S.A.

\section{Animals and diet}

Male CD1 mice (Harlan Interfauna Ibérica) weighing 30-35 g were used in the study. Mice were fed with a polyphenolfree diet for $7 \mathrm{~d}$ before the experiment to assure that the metabolites found in biological samples corresponded to the absorbed naringenin. The diet, based on Lieber et al. ${ }^{(9)}$, was isoenergetic with a multi-nutrient composition. It had the same fat content as the average 'normal' US diet, an amount recommended as 'healthy' by the Institute of Medicine, and provided sufficient vitamins and salts to prevent deficiencies in mice. The formulation contained sodium carboxymethylcellulose, which gives a semi-solid consistency that facilitates the homogeneous distribution of the components. The drinkingwater was supplemented with $\mathrm{ZnCl}_{2}(16 \cdot 3 \mathrm{mg} / \mathrm{l})$ to ensure healthy levels of this salt. Mice were housed in groups of six in cages located in temperature-controlled rooms $\left(22^{\circ} \mathrm{C}\right)$, with a dark period from 20:00 to 08:00 hours, and free access to food and water. Body weight was recorded on a daily basis. Their diet $(700 \mathrm{~g})$ was prepared twice a week.

The diet was analysed by HPLC-MS/MS to confirm the lack of naringenin and its metabolites. Extraction of the polyphenol compounds from the diet was performed in triplicate following the procedure described by Vallverdú-Queralt et al. ${ }^{(10)}$ with some modifications. Samples of $0.3 \mathrm{~g}$ were homogenised by adding $3 \mathrm{ml}$ of $80 \%(\mathrm{v} / \mathrm{v})$ ethanol in Milli-Q water acidified with $0.1 \%(\mathrm{v} / \mathrm{v})$ formic acid; after sonication for $5 \mathrm{~min}$ and centrifugation $\left(1484.7 \mathrm{~g}\right.$ at $4^{\circ} \mathrm{C}$ ) for $20 \mathrm{~min}$ (Centrifuge 5415R), the supernatant was transferred into a flask and the extraction was repeated once following the same parameters. Both supernatants were combined and evaporated under $\mathrm{N}_{2}$ flow and, finally, the residue was reconstituted with Milli-Q water $(0.1 \%$ formic acid) up to $1.2 \mathrm{ml}$, and filtered using PTFE 0.44-45 $\mu \mathrm{m}$ filters (Waters).

\section{Analytical methods}

HPLC-ESI-MS/MS analysis. All perfusion, plasma and bile samples were analysed by high-performance liquid chromatography/electrospray ionisation tandem mass spectrometry (HPLC-ESI-MS/MS). The liquid chromatograph was an Agilent series 1100 (Agilent) equipped with a thermostated autosampler and a column oven set to $30^{\circ} \mathrm{C}$. This was coupled to an API 3000 triple-quadruple mass spectrometer (PE Sciex) with a turbo ion spray source, which was used to identify and quantify the corresponding phenolic metabolites. Separation was achieved using a Luna C18 column $(50 \times 2.0 \mathrm{~mm} ; 5 \mu \mathrm{m})$ 
from Phenomenex using the following parameters: the mobile phase was $\mathrm{H}_{2} \mathrm{O}$ (A) and acetonitrile (B), with $0 \cdot 1 \%$ formic acid in both solvents. An increasing linear gradient $(\mathrm{v} / \mathrm{v})$ of $\mathrm{B}$ was used $(t(\mathrm{~min}), \% \mathrm{~B})$, as follows: $(0,5) ;(2,25)$; (10, 90); (11, $100) ;(12,100) ;(17,5) ;(20,5)$, at a constant flow rate of $0.6 \mathrm{ml} / \mathrm{min}$, and the injection volume was $20 \mu \mathrm{l}$. The turbo ion spray source was in negative mode with the following settings: capillary voltage, $-4500 \mathrm{~V}$; nebuliser gas $\left(\mathrm{N}_{2}\right), 10$ (arbitrary units); curtain gas $\left(\mathrm{N}_{2}\right), 12$ (arbitrary units); and drying gas $\left(\mathrm{N}_{2}\right)$ heated to $400^{\circ} \mathrm{C}$. The declustering potential, focusing potential, collision energy and entrance potential (Table 1) were optimised to detect phenolic compounds with the highest signals, following the method described by MartínezHuélamo and colleagues ${ }^{(11)}$. The system was controlled by Analyst version 1.4.2 software supplied by Applied Biosystems.

Naringenin and the metabolites identified in the samples, together with their retention time, limit of detection and limit of quantification, are shown in Table 1.

The calibration curves for stability and perfusion samples were prepared in phosphate buffer (PBS) pH 2, 6.2 and/or $7 \cdot 2$, as appropriate for each experiment, and were linear over the concentration ranges $0.003-4 \mu \mathrm{g} / \mathrm{ml}(r>0.998)$ for naringenin and its metabolites by HPLC-ESI-MS/MS. For the plasma and bile samples, the standards were prepared in water containing $0 \cdot 1 \%(\mathrm{v} / \mathrm{v})$ formic acid, as explained later. Metabolites were quantified by taking into account the limit of quantification. No commercial standards were available for 4-hydroxyhippuric acid and naringenin sulfate (NAR-SULF), so standards of similar structures were used for quantification: hippuric acid and unconjugated naringenin, respectively.

\section{Isotonic phosphate buffers at pH 2, 6.2 and 7.2}

The perfusion solutions (PBS) consisted of $9 \cdot 2 \mathrm{~g} / 1 \mathrm{NaCl}$, $0.036 \mathrm{~g} / 1 \mathrm{NaH}_{2} \mathrm{PO}_{4} \cdot 2 \mathrm{H}_{2} \mathrm{O}$ and $0.058 \mathrm{~g} / \mathrm{l} \mathrm{Na}_{2} \mathrm{HPO}_{4}$. The buffer $\mathrm{pH}$ was adjusted to $\mathrm{pH} 2,6 \cdot 2$ and/or 7.2 by adding orthophosphoric acid or $\mathrm{NaOH}$. The PBS contained $3.5 \mu \mathrm{g} / \mathrm{ml}$ of naringenin and $0 \cdot 1 \mathrm{mg} / \mathrm{ml}$ of phenol $\mathrm{red}^{(12)}$. The concentration of naringenin was chosen taking into account its concentration in raw tomato $(5 \mathrm{mg} / \mathrm{kg}$ approximately) and the daily ingestion of tomatoes in a Mediterranean diet $(0.5 \mathrm{~kg}$ in raw tomato, tomato juice and tomato sauce) for a $70-\mathrm{kg}$ person. The dose was then downscaled for a mouse $(30 \mathrm{~g})$. However, the estimated concentration was then increased to $3.5 \mu \mathrm{g} / \mathrm{ml}$, taking into account a potential decrease in concentration due to absorption and to be above the limit of quantification of the analytical method. The osmolality of the perfusion solutions was measured (Advanced ${ }^{\circledR}$ Model 3320 microosmometer; Advanced Instruments, Inc.).

\section{Stability and solubility studies}

The stability of naringenin in the PBS ( $\mathrm{pH} \mathrm{2,6.2} \mathrm{and} \mathrm{7.2)} \mathrm{was}$ tested at $37^{\circ} \mathrm{C}$. Six aliquots of the solutions were incubated at $37^{\circ} \mathrm{C}$, and samples were taken at $0,1,2$ and $3 \mathrm{~h}$ and stored at $-20^{\circ} \mathrm{C}$ until analysis.

The $\mathrm{pH}$-solubility profile of naringenin was determined at $37 \pm 1{ }^{\circ} \mathrm{C}$ in aqueous media at $\mathrm{pH}$ values of $1,2,5 \cdot 5,6 \cdot 2$ and 7.2. An excess of solid product was placed in a $2 \cdot 5-\mathrm{ml}$ amber vial, $2 \mathrm{ml}$ of medium was added, and the system was shaken at $37^{\circ} \mathrm{C}$ for $24 \mathrm{~h}$. After confirming the presence of undissolved material, an aliquot was removed and filtered $(0.45 \mu \mathrm{m})$ in a $37^{\circ} \mathrm{C}$ tempered vial. Then, $500 \mu \mathrm{l}$ of the filtered solution was transferred to a new vial containing $50 \mu$ l of ethanol. Finally, the concentration of naringenin in each sample was measured by HPLC. Three replicate determinations of solubility were carried out at each $\mathrm{pH}$.

\section{Perfusion experiments}

The studies were conducted following a protocol approved by the Animal Experimentation Ethics Committee of the University of Barcelona, Spain (trial no. CEEA 219/12).

Animals were monitored daily for body mass over a week to check that the diet was supplying enough nutrients and energy to maintain the growth curve ${ }^{(13)}$.

Single-pass intestinal perfusion was performed in mice according to the method described by Escribano et al. ${ }^{(8)}$, adapted for the stomach and colon. Six animals were used per biological segment (stomach, small intestine and colon), and in the case of the colon a separate group was also treated via gastric gavage with rifaximin $(50 \mathrm{mg} / \mathrm{kg} \text { per } \mathrm{d} \text { for } 5 \mathrm{~d})^{(14)}$ Rifaximin is a non-systemic antibiotic with a broad spectrum of antibacterial action used to reduce intestinal microbiota ${ }^{(15)}$. In addition, in order to investigate whether naringenin is a flavonoid with high intestinal permeability, a further batch of animals was included in the study in which a solution of

Table 1. HPLC-MS/MS settings for the phenolic compounds

\begin{tabular}{|c|c|c|c|c|c|c|c|c|}
\hline Compound & $\mathrm{RT}(\min )$ & MS/MS & $\mathrm{DP}(\mathrm{V})$ & $\mathrm{FP}(\mathrm{V})$ & $\mathrm{CE}(\mathrm{V})$ & $\mathrm{EP}(\mathrm{V})$ & $\operatorname{LOD}(\mu / \mathrm{ml})$ & $\operatorname{LOQ}(\mu / \mathrm{ml})$ \\
\hline Naringenin* & $5 \cdot 22$ & $271 \rightarrow 151$ & -50 & -190 & -30 & -11 & $2.99 \times 10^{-4}$ & $9.99 \times 10^{-4}$ \\
\hline NAR-GLU* & $4 \cdot 20$ & $447 \rightarrow 271$ & -50 & -190 & -30 & -11 & $2.51 \times 10^{-4}$ & $8.37 \times 10^{-4}$ \\
\hline NAR-SULF & $7 \cdot 14$ & $351 \rightarrow 271$ & -50 & -190 & -30 & -11 & - & - \\
\hline 3-(4-Hydroxyphenyl) propionic acid* & 2.97 & $165 \rightarrow 121$ & -30 & -200 & -20 & -10 & 0.0338 & 0.1128 \\
\hline 4-Hydroxyhippuric acid & 1.45 & $194 \rightarrow 100$ & -40 & -200 & -20 & -10 & - & - \\
\hline 3-Phenylpropionic acid* & $4 \cdot 78$ & $149 \rightarrow 105$ & -30 & -200 & -20 & -10 & 0.4400 & 14.900 \\
\hline Hippuric acid* & $2 \cdot 66$ & $178 \rightarrow 134$ & -40 & -170 & -20 & -10 & 0.0014 & 0.0049 \\
\hline (4-Hydroxyphenyl) acetic acid* & 3.03 & $151 \rightarrow 107$ & -40 & -200 & -20 & -10 & 0.0045 & 0.0151 \\
\hline
\end{tabular}

RT, retention time; DP, declustering potential; FP, focusing potential; CE, collision energy; EP, entrance potential; LOD, limit of detection; LOQ, limit of quantification; NAR-GLU, naringenin glucuronide; NAR-SULF, naringenin sulfate.

* Identified with standard. 
naproxen $(2 \cdot 15 \mu \mathrm{g} / \mathrm{ml})$, as a model of a highly permeable compound, was also assayed in the small intestine.

Mice, fed with a polyphenol-free diet for 1 week, were fasted overnight with free access to water the day before the perfusion experiment. Anaesthesia was induced by a $100 \mathrm{mg} / \mathrm{kg}$ intraperitoneal injection of ketamine (Imalgene 1000; Merial Laboratories S.A.) and $10 \mathrm{mg} / \mathrm{kg}$ of xylazin (Rompun ${ }^{\circledR} 2 \%$, Bayer S.L.). Mice were placed on a homeothermic blanket to maintain their body temperature at $37^{\circ} \mathrm{C}$. The abdomen was opened by a midline longitudinal incision, and the biliary duct was tied to avoid enterohepatic recycling. Then, the stomach (from the fundus to the pylorus), small intestine (starting from the duodenum, approximately 10$15 \mathrm{~cm}$ length) or the colon (approximately $2 \mathrm{~cm}$ length) were isolated. Two glass cannula (outer diameter $3 \mathrm{~mm}$, inner diameter $2 \mathrm{~mm}$; Duran ${ }^{\circledR}$, Vidrafoc) were inserted at the proximal and distal ends of the segment. The segment was gently rinsed with $\mathrm{PBS} \mathrm{pH} 2,6 \cdot 2$ or 7.2 (respectively) to remove the contents until the outlet solution appeared clear. Amber latex tubing (inner diameter $3 \mathrm{~mm}$ ) was then connected to the inlet cannula, and a perfusion pump (Minipuls3, M312 model; Gilson, Inc.) was placed between the perfusate reservoir and the inlet cannula. Saline was dripped onto the surgical area, which was then covered with gauze to avoid dehydration. The experiment started by delivering the perfusion solution containing naringenin and phenol red at a flow rate of $0.20 \mathrm{ml} / \mathrm{min}(0 \cdot 16 \mathrm{ml} / \mathrm{min}$ for the stomach) to the tubes and intestinal segment. The outflow perfusate was collected in 1.5-ml amber eppendorfs at 10-min intervals for $60 \mathrm{~min}$. The first sample was taken $5 \mathrm{~min}$ after the start of continuous outlet flow from the intestine and through the distal cannula. Samples were centrifuged $(7516.3 \mathrm{~g}$ for $10 \mathrm{~min}$ at $4^{\circ} \mathrm{C}$ ) (Centrifuge $5415 \mathrm{R}$ ) and $650 \mu \mathrm{l}$ aliquots of the supernatant were spiked with $50 \mu \mathrm{l}$ of ethanol to avoid precipitation of naringenin at lower temperatures and were then stored frozen $\left(-80^{\circ} \mathrm{C}\right)$ until analysis. At $60 \mathrm{~min}$, a blood sample was obtained via cardiac puncture, the gallbladder was collected and the length of the intestinal segment was measured. Bile samples from four to six mice were pooled and stored with all samples at $-80^{\circ} \mathrm{C}$ until analysis by HPLC-MS/MS.

\section{Solid-phase extraction of polyphenols from plasma and bile samples}

On the day of analysis by HPLC-ESI-MS/MS, plasma and bile samples were defrosted and centrifuged $(7516 \cdot 3 \boldsymbol{g}$ for $10 \mathrm{~min}$ at $\left.4^{\circ} \mathrm{C}\right)$. An exact volume of supernatants was transferred into an amber eppendorf and diluted to $1 \mathrm{ml}$ with water containing $0 \cdot 1 \%(\mathrm{v} / \mathrm{v})$ formic acid. An Oasis ${ }^{\circledR}$ HLB 30- $\mu \mathrm{m}(30 \mathrm{mg})$ 96-well plate (Waters) was activated by consecutively adding $1 \mathrm{ml}$ of methanol and $1 \mathrm{ml}$ of $1.5 \mathrm{M}$ formic acid in water. The samples were loaded onto the plate, and cleaned up by adding $1 \mathrm{ml}$ of $1.5 \mathrm{M}$ formic acid, followed by $1 \mathrm{ml}$ methanol: water ratio (5:95). Phenolic metabolites were then eluted with $1 \mathrm{ml}$ of methanol acidified with $0 \cdot 1 \%$ formic acid (v/v). The eluted fraction was evaporated to dryness (Techne) at room temperature under a stream of $\mathrm{N}_{2}$, and reconstituted with $100 \mu \mathrm{l}$ of water containing $0 \cdot 1 \%(\mathrm{v} / \mathrm{v})$ formic $\operatorname{acid}^{(16)}$.
All procedures were performed in a darkened room with a red safety light to avoid oxidation of the analytes.

\section{Data analysis}

Peff were calculated from the steady-state concentrations of naringenin in the collected perfusate, according to equation $1^{(17)}$ after correcting the outlet concentration according to the phenol red method ${ }^{(18,19)}$ (equation 2).

$$
\text { Peff }=\frac{-\phi_{\text {in }}}{2 \pi R L} \operatorname{Ln} \frac{C_{\text {in }}}{C_{\text {out.cor }}}
$$

where $\phi_{\text {in }}$ is the flow rate $(0.20$ and $0.16 \mathrm{ml} / \mathrm{min}$ for the stomach), $C_{\text {in }}$ and $C_{\text {out.cor }}$ are the respective inlet and corrected outlet steady-state concentrations (equation 2 ), $R$ is the radius of the mouse gastrointestinal segment $(0 \cdot 1 \mathrm{~cm}$ for small intestine ${ }^{(20,21)}, 0.14 \mathrm{~cm}$ for colon $\left.{ }^{(22)}\right)$; and in the case of the stomach, $R$ was calculated assuming it to have the shape of a cylinder with a volume of $0.4 \mathrm{ml}^{(23)}$, and according to equation $3, L$ is the length of gastrointestinal segment after completion of the perfusion experiment.

$$
C_{\text {out.cor }}=C_{\text {out }} \times \frac{\mathrm{CPR}_{\text {in }}}{\mathrm{CPR}_{\text {out }}}
$$

where $C_{\text {out }}$ is the concentration of naringenin in the perfusate at the specified time interval, $\mathrm{CPR}_{\mathrm{in}}$ is the phenol red concentration in the inlet buffer solution and $\mathrm{CPR}_{\text {out }}$ is the phenol red concentration at the specific time interval.

$$
R=\sqrt{\frac{0.4}{\pi L}}
$$

The Peff was determined in four to five mice at between four and six steady-state outlet concentrations, and expressed as means and standard deviations.

The percentage absorption (\%abs) was calculated according to equation $4^{(24)}$ and the results were expressed as means and standard deviations.

$$
\% \text { abs }=\left[1-e^{\frac{2 \pi R L}{\phi_{\text {in }}} \text { Peff }}\right] \times 100
$$

Naringenin and metabolites were analysed in the collected perfusate at 10-min intervals. For the main metabolites, a linear trapezoidal method was used to calculate the area under the lumen concentration curve AUC (normalised by the inlet perfusion concentration of naringenin) from time zero until the last sample, using the WinNonlin ${ }^{\circledR}$ software version 6.3 (2012 Certara, L.P.). AUC values were expressed in min.

In perfusate $(60 \mathrm{~min})$, plasma and bile samples, the percentage of each metabolite with respect to the inlet naringenin perfusion solution $\left(C_{\mathrm{in}}\right)$ was calculated according to equation 5 :

$$
\% C_{\text {met }}=\left(C_{\text {met }} \times 100\right) / C_{\text {in }}
$$

where $C_{\text {met }}$ is the concentration of each metabolite in the sample. 
Table 2. Naringenin effective permeability coefficients (Peff) and percentage of absorption in each gastrointestinal segment (Mean values and standard deviations)

\begin{tabular}{lllllll}
\hline & \multicolumn{2}{c}{$\begin{array}{c}\text { Peff } \\
\left(\times 10^{-4} \mathrm{~cm} / \mathrm{s}\right)\end{array}$} & & \multicolumn{2}{c}{ Absorption $(\%) \ddagger$} \\
\cline { 2 - 3 } & Mean & SD & & Mean & SD & $n$ \\
\hline Stomach & 3.04 & 0.89 & & 23.69 & 5.54 & 4 \\
Small intestine & 7.80 & 1.54 & & $76.28^{\star}$ & 6.78 & 4 \\
Colon & $5.49 \dagger$ & 1.86 & & $28.01 \dagger$ & 7.37 & 5 \\
Colon (rifaximin) & 2.26 & 0.96 & & 11.18 & 4.46 & 5 \\
\hline
\end{tabular}

$n$, Number of replicates.

${ }^{*}$ Mean value was significantly different from that for colon segments $(P<0.05$; Student's $t$ test).

†Mean value was significantly different from that for rifaximin-treated colon segments $(P<0.05$; Student's $t$ test).

$\ddagger$ Absorption for $10 \mathrm{~cm}$ in the small intestine and $2 \mathrm{~cm}$ in the colon

\section{Statistical analysis}

The estimated concentrations at a range of times in the stability study were compared using a parametric statistical assay (ANOVA), followed by a Scheffe test, where appropriate.

The Peff and percentages of absorption in the small intestine and colon were compared by means of Student's $t$ test after verifying their normal distribution (Kurtosis normality of residuals) and the homogeneity of variance (modified Levene equal - variance test). The same test was applied to study the influence of antibiotic treatment on the absorption of naringenin in the colon (comparison made between the control group and the group treated with rifaximin) (Table 2).

The amount of NAR-GLU and NAR-SULF (expressed as AUC) in the lumen in each gastrointestinal segment, and their percentages $(60 \mathrm{~min})$ in the lumen, bile and plasma in the two groups were compared using the Mann-Whitney $U$ test, after rejecting their normal distribution and the homogeneity of variance (Fig. 2 and Table 3, respectively).

The mean AUC of the colonic metabolites in the control and rifaximin-treated groups (Table 4) was compared by the Kruskal-Wallis one-way ANOVA followed by the KruskalWallis multiple-comparison $Z$ test, after rejecting their normal distribution and the homogeneity of variance. Moreover, to determine differences in metabolites between the two groups (control and rifaximin-treated), the data were analysed using Student's $t$ test.

In all cases, the level of significance was 5\% $(P<0.05)$ and NCSS97 statistical software was used.

\section{Results}

\section{Suitability of the diet}

Three aliquots of the polyphenol-free diet were analysed by HPLC-ESI-MS/MS after polyphenol extraction, modifying the method used by Vallverdú-Queralt et al. ${ }^{(25)}$. The chromatograms showed the absence of naringenin, naringenin-metabolites and other polyphenols. The body weight of the animals on the polyphenol-free diet remained steady.

\section{Stability and solubility studies}

Fig. 1(a)-(c) shows the naringenin stability at the $\mathrm{pH}$ of the absorption studies. Naringenin was stable at $\mathrm{pH} 2$ for $3 \mathrm{~h}$ at $37^{\circ} \mathrm{C}(P=0.716)$, whereas at $\mathrm{pH} 6.2$ and 7.2 it was only stable for $2 \mathrm{~h}$.

Fig. 1(d) shows the pH-solubility profile of naringenin in the $\mathrm{pH}$ range studied. The solubility increased with the $\mathrm{pH}$ according to the acidic nature of the compound, and ranged between $22.88(\mathrm{SD} 0.60) \mu \mathrm{g} / \mathrm{ml}$ at $\mathrm{pH} 1$ and 46.86 (SD $0 \cdot 38) \mu \mathrm{g} / \mathrm{ml}$ at $\mathrm{pH} 7 \cdot 2$

In situ absorption studies of naringenin in the stomach, small intestine and colon

The Peff and the absorption percentage in mice using an in situ single-pass perfusion technique for each intestinal segment are shown in Table 2. Also included are the results of naringenin absorption in the colon after treatment with the antibiotic rifaximin.

The highest permeability was obtained in the small intestine $\left(\right.$ Peff $\left.=7.80(\mathrm{sD} 1.54) \times 10^{-4} \mathrm{~cm} / \mathrm{s}\right)$, followed by the large intestine $\left(\right.$ Peff $\left.=5.49 \quad(\mathrm{SD} 1.86) \times 10^{-4} \mathrm{~cm} / \mathrm{s}\right)$, while the lowest was in the stomach (Peff $=3.04($ SD 0.89$\left.) \times 10^{-4} \mathrm{~cm} / \mathrm{s}\right)$.

The percentage of naringenin was highest in plasma samples from a small intestine perfusion assay (29.10 (SD 6.72)\%), being lower in the colon samples (0.68 (SD 0.19)\%), while none was found in the stomach samples. Naringenin was not excreted into the bile intact.

\section{Regional differences in naringenin metabolism}

The mean areas and standard deviations under the lumen concentration curve (AUC) from time zero until the last sample of the metabolites NAR-GLU and NAR-SULF are shown in Fig. 2. Results of the statistical analysis are also

Table 3. Percentage of naringenin sulfate (NAR-SULF) and naringenin glucuronide (NAR-GLU) in perfusate $(60 \mathrm{~min})$, bile and plasma in the three gastrointestinal segments

(Mean values and standard deviations)

\begin{tabular}{|c|c|c|c|c|}
\hline & \multicolumn{2}{|c|}{ NAR-SULF (\%) } & \multicolumn{2}{|c|}{ NAR-GLU (\%) } \\
\hline & Mean & SD & Mean & SD \\
\hline \multicolumn{5}{|l|}{ Stomach } \\
\hline Perfusate & $0.007^{*} \dagger$ & 0.004 & $0.03^{*} \dagger$ & 0.00 \\
\hline Bile & ND & & ND & \\
\hline Plasma & ND & & $9 \cdot 260^{*} \dagger$ & $2 \cdot 20$ \\
\hline \multicolumn{5}{|c|}{ Small intestine } \\
\hline Perfusate & $84.00 \dagger$ & $12 \cdot 14$ & $8.40 \dagger$ & $5 \cdot 67$ \\
\hline Bile & ND & & ND & \\
\hline Plasma & ND & & $22 \cdot 86 \dagger$ & 8.38 \\
\hline \multicolumn{5}{|l|}{ Colon } \\
\hline Perfusate & 3.66 & 1.75 & 0.16 & 0.03 \\
\hline Bile & ND & & ND & \\
\hline Plasma & ND & & 2.990 & $1.61 C$ \\
\hline
\end{tabular}

ND, not detected.

* Mean value was significantly different from that for small intestine segments $(P<0.05$; Mann-Whitney $U$ test $)$.

† Mean value was significantly different from that for colon segments $(P<0.05$; Mann-Whitney $U$ test). 
Table 4. AUC of the metabolites in colonic lumen in control mice and in mice treated with rifaximin (Mean values and standard deviations)

\begin{tabular}{|c|c|c|c|c|c|c|c|c|}
\hline & \multicolumn{8}{|c|}{ AUC (min) } \\
\hline & \multicolumn{2}{|c|}{ NAR-GLU } & \multicolumn{2}{|c|}{ NAR-SULF } & \multicolumn{2}{|c|}{$\begin{array}{l}\text { 4-Hydroxyhippuric } \\
\text { acid }\end{array}$} & \multicolumn{2}{|c|}{ Hippuric acid } \\
\hline & Mean & SD & Mean & SD & Mean & SD & Mean & SD \\
\hline Control & $0.079^{\star}$ & 0.027 & $2 \cdot 616$ & $1 \cdot 347$ & $0.219^{\star} \dagger$ & 0.072 & $0.168^{*}$ & 0.012 \\
\hline Treated & $0.042^{*}$ & 0.044 & $4 \cdot 207$ & $2 \cdot 326$ & $0.072^{\star} \ddagger$ & 0.036 & $0.062^{*}$ & 0.001 \\
\hline
\end{tabular}

included. It should be noted that the units of the area are min (and the concentration units cancel out) due to the normalisation of the metabolite concentration by the inlet concentration of naringenin. The highest significant $(P<0.05)$ levels of metabolites were observed in the small intestine, followed by the colon. In both intestinal regions, the main metabolite was NAR-SULF. In the stomach, higher levels of NAR-GLU $v$. NAR-SULF were obtained.

In Table 3, the mean percentages and standard deviations of each phase II metabolite at $60 \mathrm{~min}$ in perfusate, bile and plasma are shown. In general, a higher percentage of NAR-GLU was observed in plasma $v$. perfusate for the three gastrointestinal segments. NAR-SULF was not detected in plasma, and neither metabolite was found in the bile.

\section{Effects of the antibiotic treatment on the absorption and metabolism of naringenin}

Effects of the antibiotic treatment on naringenin absorption are shown in Table 2. As can be observed, the pretreatment with rifaximin reduced $(P<0.05)$ the Peff of naringenin in the large intestine $\left(5.49(\mathrm{SD} 1.86) \times 10^{-4} \mathrm{~cm} / \mathrm{s} \quad v .2 .26\right.$
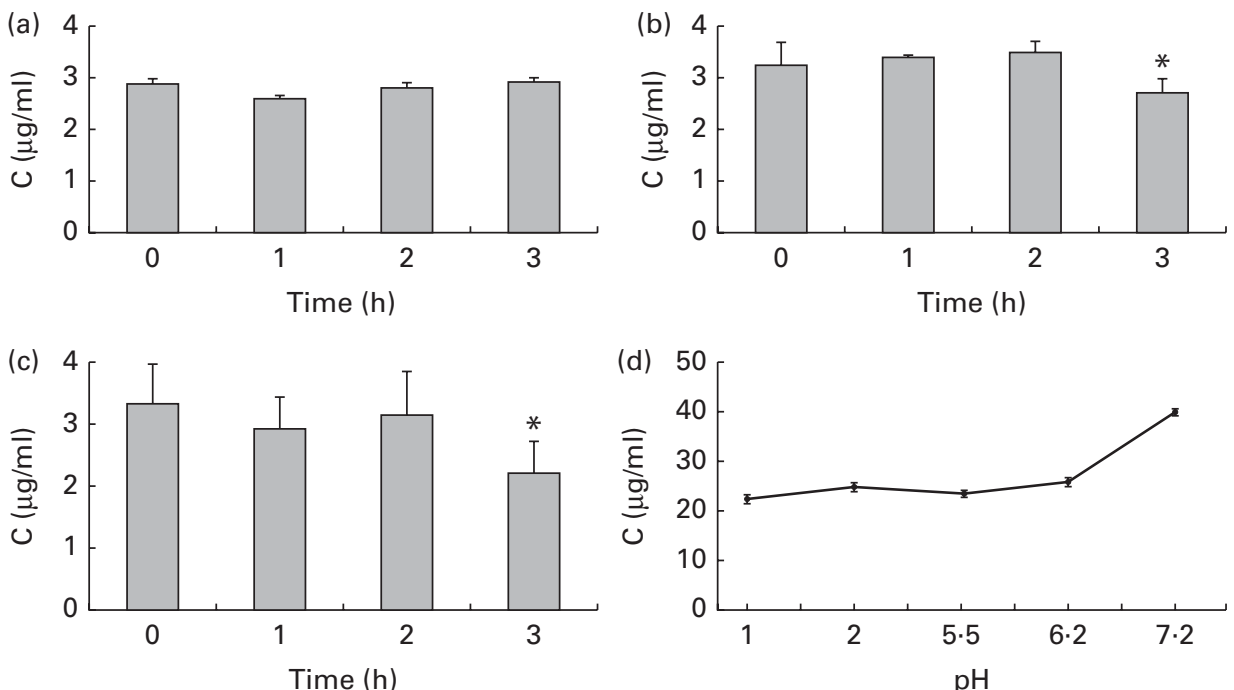

Fig. 1. Mean concentrations of naringenin (C) in the perfusion buffers $v$. time in the stability assay at $\mathrm{pH} 2(\mathrm{a}), \mathrm{pH} 6.2(\mathrm{~b})$ and $\mathrm{pH} 7.2$ (c); and the $\mathrm{pH}$-solubility profile of naringenin (d). Values are means and standard deviations represented by vertical bars. ${ }^{*}$ Mean value is significantly different from that at time $0(P<0.05)$. 


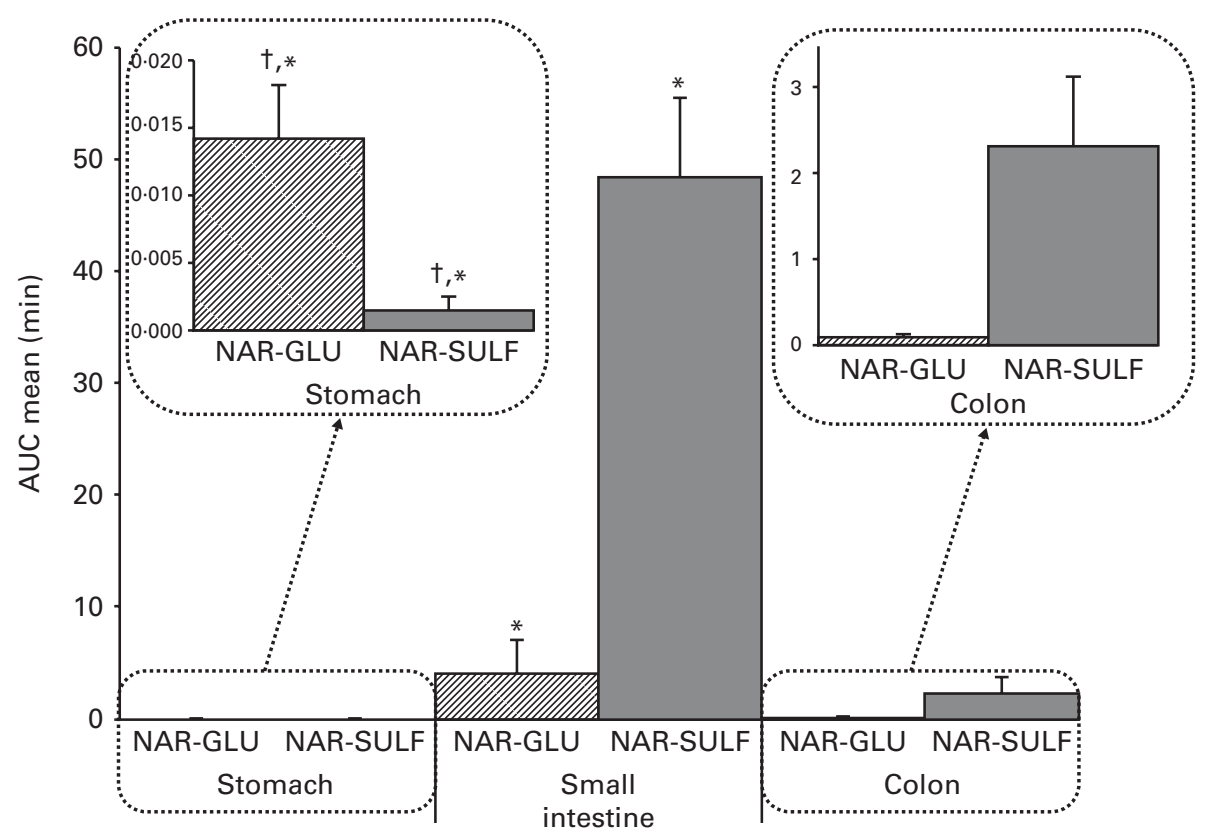

Fig. 2. Area under the lumen concentration curve of naringenin glucuronide (NAR-GLU) and naringenin sulfate (NAR-SULF) in stomach, small intestine and colon ( $n$ 6, 4 and 6, respectively). Values are means and standard deviations represented by vertical bars. *Mean value is significantly different from that of colon $(P<0.05)$. †Mean value is significantly different from that of small intestine $(P<0.05)$.

at $\mathrm{pH} 2,6.2$ and $7 \cdot 2$ for the time required to perform the permeation experiments.

According to the $\mathrm{BCS}^{(5)}$, a compound is considered highly soluble when the highest dose strength is soluble in $250 \mathrm{ml}$ or less of aqueous media over the $\mathrm{pH}$ range of $1-7 \cdot 5$. As naringenin is a dietary compound, it cannot be treated as a drug - in terms of dose strength - when testing for solubility. However, according to the values obtained $(<0.0047 \%)$, it can be considered as a compound with low solubility. Nonetheless, if we consider the lowest value of solubility at $37^{\circ} \mathrm{C}$, which occurs under the most acidic conditions $(22.88$ ( $\mathrm{SD} 0.60) \mu \mathrm{g} / \mathrm{ml}$ at $\mathrm{pH} 1$ ), it is still higher than the concentration of naringenin in food sources. Therefore, the higher solubility of naringenin at physiological $\mathrm{pH}$ values is not expected to be a limiting factor in the absorption of naringenin after its oral ingestion.
In situ absorption studies of naringenin in the stomach, small intestine and colon

The Peff obtained in the three gastrointestinal regions showed that more naringenin was absorbed in the small intestine than in the stomach and colon $\left(7.80(\mathrm{SD} 1.54) \times 10^{-4} \mathrm{~cm} / \mathrm{s} v .3 .04\right.$ $(\mathrm{SD} 0.89) \times 10^{-4} \mathrm{~cm} / \mathrm{s}$ and $5.49(\mathrm{SD} 1.86) \times 10^{-4} \mathrm{~cm} / \mathrm{s}$, respectively). However, this parameter did not differ significantly between the small and large intestines. In the present study, the permeability of naproxen, as a model of a highly permeable compound, was also assayed in the small intestine. The Peff obtained $\left(6.39(\mathrm{sD} 1.23) \times 10^{-4} \mathrm{~cm} / \mathrm{s}\right)$ was similar to that for naringenin $\left(7.80(\mathrm{SD} 1.54) \times 10^{-4} \mathrm{~cm} / \mathrm{s}\right)$, indicating that naringenin permeability was high and maintained throughout the small intestine and colon. Both compounds (naringenin and naproxen) have a low molecular weight

Table 5. Percentage (\%) of phase II and microbial metabolites in the bile (pooled sample) and plasma from colon perfusion experiments in control mice and in mice treated with rifaximin

(Mean values and standard deviations; $n 5-6$ )

\begin{tabular}{|c|c|c|c|c|c|c|}
\hline & & & \multicolumn{4}{|c|}{ Plasma } \\
\hline & \multicolumn{2}{|c|}{ Bile } & \multicolumn{2}{|c|}{ Control } & \multicolumn{2}{|c|}{ Treated } \\
\hline & Control & Treated & Mean & SD & Mean & SD \\
\hline NAR-GLU & ND & ND & 2.99 & $1 \cdot 61$ & $0.49^{*}$ & 0.31 \\
\hline NAR-SULF & ND & ND & ND & & 0.70 & 0.20 \\
\hline 3-(4-Hydroxyphenyl) propionic acid & $7 \cdot 43$ & $8 \cdot 30$ & $10 \cdot 34$ & $3 \cdot 14$ & $13 \cdot 63$ & $4 \cdot 18$ \\
\hline 4-Hydroxyhippuric acid & 41.97 & $20 \cdot 01$ & $1 \cdot 12$ & 0.76 & 1.49 & 0.70 \\
\hline Hippuric acid & 3.28 & $1 \cdot 10$ & 1.87 & 0.80 & 1.45 & 0.44 \\
\hline 3-Phenylpropionic acid & $48 \cdot 11$ & $125 \cdot 68$ & $59 \cdot 77$ & $15 \cdot 39$ & $60 \cdot 39$ & $16 \cdot 40$ \\
\hline (4-Hydroxyphenyl) acetic acid & $16 \cdot 88$ & 2.54 & 1.97 & 0.91 & $3.66 \dagger$ & 0.75 \\
\hline
\end{tabular}

NAR-GLU, naringenin glucuronide; ND, not detected; NAR-SULF, naringenin sulfate.

* Mean value was significantly different from that of the control group $(P<0.05$; Mann-Whitney $U$ test).

† Mean value was significantly different from that of the control group $(P<0.05$; Student's $t$ test). 
and high lipophilicity (expressed as $\log P)(272 \cdot 25 \mathrm{~g} / \mathrm{mol}$ and $\log P=2 \cdot 84^{(26)}$ v. $230 \cdot 26 \mathrm{~g} / \mathrm{mol}$ and $\log P=2.52^{(4)}$ for naringenin and naproxen, respectively), properties that favour intestinal absorption. Fagerholm and colleagues ${ }^{(27)}$ and Masaoka et al. $^{(22)}$ found similar results for naproxen in rats: the decrease in Peff from the proximal to the distal intestine was less pronounced for the more lipophilic compounds, and the permeability was similar in the jejunum, ileum and colon. Based on these results, although naringenin has been described as a substrate of P-glycoprotein and MRP-2 (efflux proteins) and MRP-1 (influx) ATP-dependent transporters ${ }^{(4)}$, the main route of absorption for naringenin is by passive diffusion. However, this can lead to pharmacokinetic interactions with other drug/compound substrates of the same transporters, as described by Takanaga et al. ${ }^{(28)}$, Bailey et al. ${ }^{(29)}$ and Le Goff et $a l{ }^{(30,31)}$. In this respect, naringenin is also an inhibitor of the isoform CYP3A4, which plays an important role in the metabolism of many drugs ${ }^{(32)}$.

Regarding permeability in the stomach, the Peff values were not comparable with those for the intestine because of the different flow rate used in the experiments $(0.16 v \cdot 0 \cdot 2 \mathrm{ml} / \mathrm{min}$, respectively). Komiya et $a l^{(24)}$ demonstrated that the permeability and percentage absorbed could change due to differences in residence time in the organ caused by the flow rate. The fraction absorbed is expected to be higher with lower flow rates, because as residence time increases, the molecule has more opportunities to be absorbed. However, the results obtained for naringenin show that the Peff in the stomach was lower than in the small intestine and colon, although still significant.

As shown in Table 2, the absorption percentage did not always correspond to the Peff. For example, one could expect a higher absorption percentage in the colon according to the Peff. This variance can be attributed to the different length segments used in the experiments: $1.5-2 \mathrm{~cm}$ in the colon, $10-15 \mathrm{~cm}$ in the small intestine and $1.1-1.35 \mathrm{~cm}$ in the stomach (and in this case, assuming a cylindrical shape). Parameter $L$ (equations 1 and 4) affects the percentage absorbed more than the Peff. These results are consistent with Komiya et $a$. $^{(24)}$, who studied the fraction absorbed and the Peff of seven steroids in rats using different lengths of the intestine.

\section{Regional differences in naringenin metabolism}

With the aim of studying the intestinal first-pass metabolism, lumen samples, bile and blood $(60 \mathrm{~min})$ taken from the stomach, small intestine and colon assays were examined for the presence of metabolites. The main phase II metabolites found in the intestinal perfusion samples were mainly NARSULF, followed by NAR-GLU $(P<0 \cdot 05)$.

The mean AUC values for these metabolites in each gastrointestinal segment are shown in Fig. 2. Considering the total conjugates excreted, their levels according to site were ranked: small intestine $>$ colon $>$ stomach. The AUC for NAR-SULF was 48.314 (SD 7.166) min, 2.616 (SD 1.347) min and 0.001 (SD 0.002) min, for the small intestine, colon and stomach, respectively. Chen et al. ${ }^{(33)}$ studied the distribution of human gastrointestinal sulfotransferases and demonstrated that the small intestine has higher sulfation activity than either the stomach or the colon, which is consistent with the present study results. Similar behaviour was observed for the uridine $5^{\prime}$-diphospho-glucoronosyltransferases (UDP glucuronosyltransferases), whose activity was also higher in the small intestine (AUC of NAR-GLU 4.072 (SD 2.948) min v. 0.079 ( $\mathrm{SD} 0.027) \mathrm{min}$ and $0.014(\mathrm{SD} 0.006) \mathrm{min}$, for the colon and stomach, respectively). Gregory et al. ${ }^{(34)}$ and $\mathrm{Xu}$ et al. $^{(1)}$ obtained similar results.

In the lumen, the main metabolites excreted were sulfates, as in human subjects ${ }^{(7)}$. Sulfation is generally a higher-affinity, lower-capacity pathway than glucuronidation ${ }^{(33)}$. In the present study, according to the low concentration assayed, higher sulfate production was expected. Both metabolites (sulfates and glucuronides) started to appear $5 \mathrm{~min}$ after starting the perfusion, but at different levels (Fig. 3). At $60 \mathrm{~min}$, the mean percentage of NAR-SULF was 84.00 (SD 12.14) $\%$ in the small intestine, whereas for glucuronide it was 8.40 (SD 5.67) \% (Table 3). In other words, the levels of NAR-SULF from 10 to 60 min (steady levels) were in the same order of magnitude as the parent compound, while those of NAR-GLU were lower (with a steady profile from approximately $30 \mathrm{~min}$ ).

Only NAR-GLU (and not NAR-SULF) was found in the plasma and the highest percentage was obtained in the plasma of small intestine assays (22.86 (SD 8.38)\%). Neither NAR-GLU nor NAR-SULF was detected in the bile. Contradictory results have been found by other authors: Fang et al. ${ }^{(35)}$, who studied the bioavailability of naringenin and naringin after oral administration in rats, reported a far higher plasma
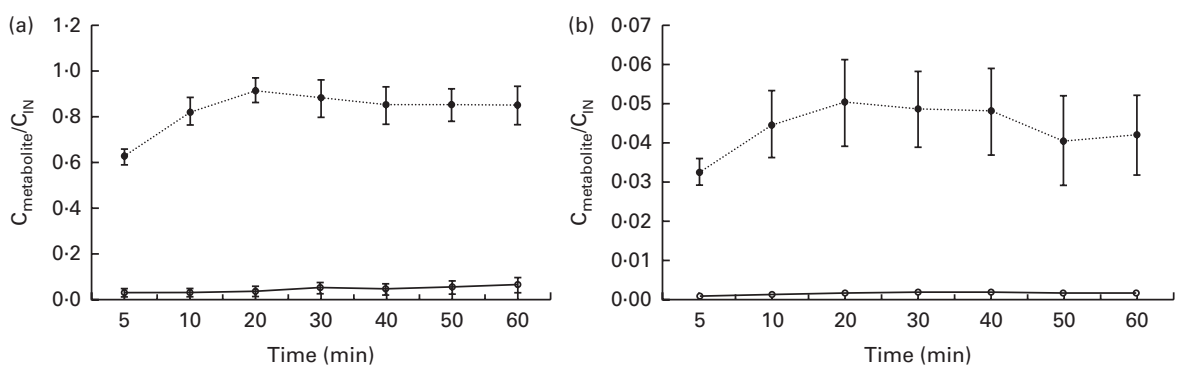

Fig. 3. Concentration of metabolites $\left(\mathrm{C}_{\text {metabolite }}\right)$ naringenin glucuronide $(-)$ and naringenin sulfate (....) normalised to the inlet concentration $\left(\mathrm{C}_{\mathrm{IN}}\right)$ of naringenin $v$. time in the small intestine (a) and colon (b). Values are means, with their standard errors represented by vertical bars. 
profile of NAR-SULF than of NAR-GLU (5.4 times higher in terms of AUC), while Xu et $a l^{(1)}$ found significant amounts of NAR-GLU in rat bile, more than double that of intestinal lumen samples, using a similar intestinal perfusion technique.

According to the present study results and the high levels of sulfates found in the lumen with respect to the plasma and bile, we suggest that naringenin sulfation in mice occurs mainly in the enterocytes rather than in the liver. Further research is required to study the sulfotransferases involved in the intestinal metabolism of naringenin. Regarding glucuronisation, the process seems to take place in both the intestinal cells and the liver, and the isoform UDP glucuronosyltransferases 1A localised in the endoplasmic reticulum of the enterocytes plays an important role in the first-pass metabolism of naringenin and other polyphenols ${ }^{(36)}$.

\section{Effect of the antibiotic treatment on the absorption and} metabolism of naringenin

The aim of this part of the work was to study the influence of the microbiota on the absorption and metabolism of naringenin
As can be observed in Table 2, pretreatment with rifaximin reduced the permeability of naringenin in the large intestine $(P<0.05)$. This reduction in absorption is in accordance with the lower percentage of NAR-GLU observed in plasma (Table 5) of the rifaximin-treated mice compared to the control group (0.49 (SD 0.31)\% v. 2.99 (SD 1.61)\%, respectively) as well as a decrease in the percentage of naringenin in plasma (from 0.68 (SD 0.19) \% to 0.37 (SD 0.09) \% for treated mice). In contrast, plasma NAR-SULF increased in rifaximin-treated mice, although the percentage was quite low $(0.7(\operatorname{sD~} 0 \cdot 2) \%)$

In the colonic perfusion samples (Table 4), the amounts of phase II metabolites (NAR-GLU and NAR-SULF) did not differ significantly $(P>0.05)$ between the two groups. As they are mainly intestinal and hepatic metabolites, differences were not expected.

Regarding the microbial metabolites, naringenin can be metabolised to a wide range of low-molecular-weight aromatic acids such as phloroglucinol, 3-(4-hydroxyphenyl) propionic acid, (4-hydroxyphenyl) acetic acid, 3-phenylpropionic acid, 4-hydroxyhippuric acid and hippuric acid ${ }^{(37-39)}$. Based on the present study results (Tables 4 and 5), a scheme of the colonic metabolic pathway of naringenin is

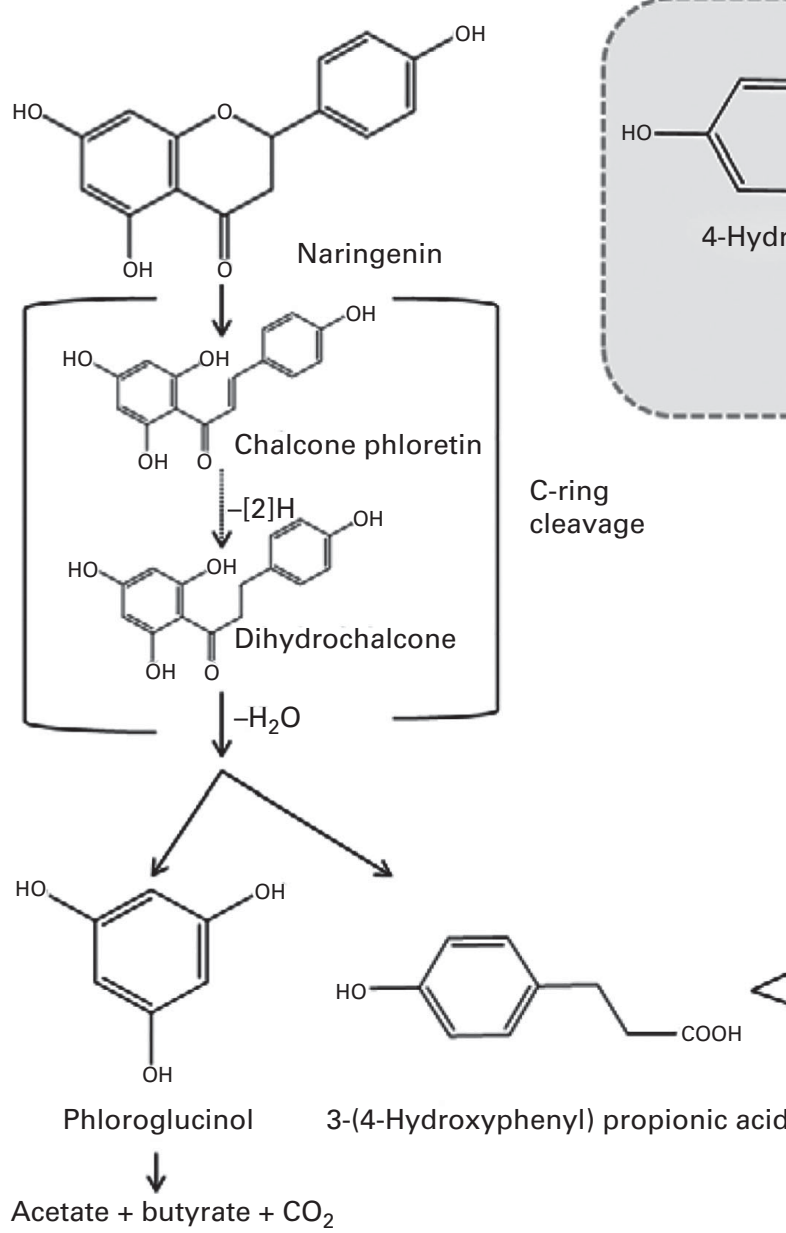

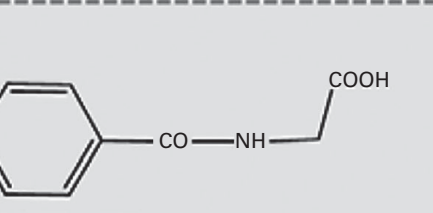

-Hydroxyhippuric acid

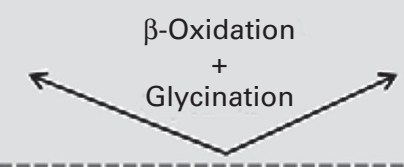

Hippuric acid
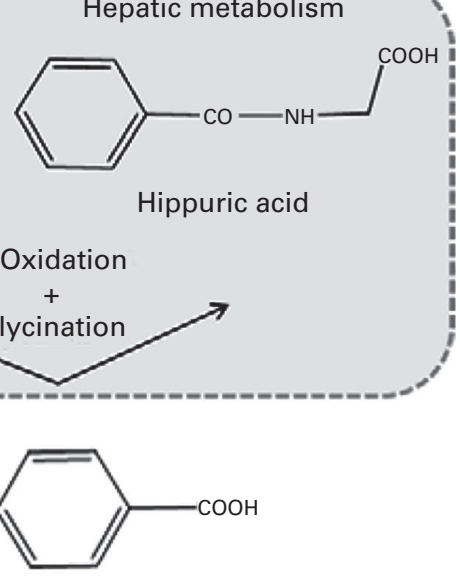

Benzoic acid

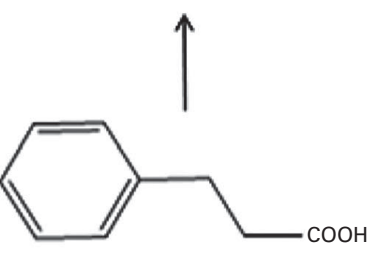

3-Phenylpropionic acid

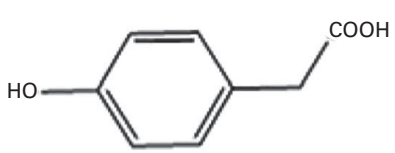

(4-Hydroxyphenyl) acetic acid

Fig. 4. Main metabolic pathway tentatively proposed for the colonic metabolism of naringenin ${ }^{(37-39,42)}$. 
proposed in Fig. 4. As shown, 3-phenylpropionic acid may be biotransformed to benzoic acid, absorbed (by the monocarboxylic acid transporter 1) and subjected to glycination and $\beta$-oxidation in the liver, yielding hippuric acid and 4-hydroxyhippuric acid ${ }^{(37,40-43)}$. This pathway justifies the presence of 4-hydroxyhippuric acid and hippuric acid in the bile and plasma. On the other hand, the presence of these metabolites in the colonic perfusates (Table 4) under our experimental conditions (bile duct tied) could be explained by intestinal secretion. Although hippuric acid can be derived from sources other than polyphenols, such as aromatic amino acids ${ }^{(36)}$, the relatively high levels obtained may be associated with the dose of naringenin administered. Phloroglucinol was not detected in the present study and is rarely recovered as a final metabolite, as it can be degraded into acetate, butyrate and $\mathrm{CO}_{2}^{(38)}$.

There was a lack of hydroxyphenyl propionic acids and hydroxyphenyl acetic acids (intermediate metabolites) in the perfusate samples, although we detected and quantified 3-(4-hydroxyphenyl) propionic acid, 3-phenylpropionic acid and (4-hydroxyphenyl) acetic acid in the plasma and bile. This absence could be attributed to rapid intestinal absorption of the phenolic acids after their formation. Konishi \& Kobayashi ${ }^{(44)}$ reported the permeation of 3-(3,4-dihydroxyphenyl) propionic acid via the paracellular pathway and of 3-(3hydroxyphenyl) propionic acid by the monocarboxylic acid transporter. No significant differences in 3-(4-hydroxyphenyl) propionic acid in the plasma and bile were found between the two experimental groups (control and treated with rifaximin), in contrast with the derived metabolites (3-phenylpropionic acid and (4-hydroxyphenyl) acetic acid) in bile. The high percentage of 3-phenylpropionic acid found in the samples was striking, although it can be considered as reasonable, since its presence in biological samples may be attributed to the bacterial metabolic action of dietary polyphenols as well as dietary phenolic amino acids such as phenylalanine and tyrosine $e^{(45,46)}$

In principle, we would expect higher levels of microbial metabolites in control animals than in those treated with rifaximin, but no significant differences were observed between the two groups. These results suggest that the antibiotic treatment did not totally eliminate the intestinal microbiota but probably altered it. Gao and colleagues ${ }^{(47)}$ reported that rifaximin alters the bacterial population in the ileum of rats, leading to a relative abundance of Lactobacillus species. In the present study, an increase in 3-phenylpropionic acid in the bile and in (4-hydroxyphenyl) acetic acid in the plasma of rifaximin-treated mice was observed.

On the other hand, taking into account the relatively high percentage of microbial metabolites found in the biological samples, it is of great interest to consider their possible contribution to the human health benefits attributed to the parent compound naringenin. In this context, microbial metabolites such as hydroxylated 3-phenylpropionic acids and hydroxylated phenylacetic acids retain antioxidant properties, while others such as (4-hydroxyphenyl) acetic acid are reported to be more effective than their parent compounds (rutin and quercetin) in inhibiting platelet aggregation in vitro ${ }^{(44)}$.
Miene and colleagues ${ }^{(48)}$ also report anti-inflammatory activity of (3,4-dihydroxyphenyl) acetic acid and 3-(3,4-dihydroxyphenyl) propionic acid in human colon cells. It could be extrapolated from these results that if naringenin were ingested in the diet, the food matrix would favour the production of microbial metabolites, since prebiotic and probiotic compounds can stimulate intestinal fermentation and the activity of the colonic microbiota ${ }^{(36)}$. The matrix could even bind or occlude naringenin, thereby hampering its absorption and increasing the residence time of unabsorbed naringenin in the gut and making it more available for the activity of the microbiota.

\section{Conclusions}

The present study results show that naringenin is a highly permeable compound (comparable to naproxen) and is absorbed throughout the gastrointestinal tract, but mainly in the small intestine and by a passive diffusion mechanism. Neither its permeability nor solubility at physiological $\mathrm{pH}$ values are expected to be a limiting factor in the bioavailability of naringenin after its dietary ingestion.

The low oral bioavailability of naringenin can be attributed to a high intestinal first-pass effect, and, to a lesser degree, to hepatic metabolism. The high intestinal first-pass metabolism of naringenin leads to a high level of phase II metabolites (mainly naringenin- $O$-sulfate in the intestinal lumen), which - due to an enterohepatic cycle - would extend the presence of naringenin within the organism. The main sulfation and glucuronidation activity was found in the small intestine, followed by the colon and stomach. The treatment with rifaximin $(50 \mathrm{mg} / \mathrm{kg})$ led to a decrease in the absorption of naringenin but not to a significant reduction in any of the microbial metabolites formed.

According to the present study results, the health effects associated with dietary naringenin seem to be more closely related to the high level of bioactive metabolites formed than to its bioavailability. However, further pharmacodynamics and metabolic studies are needed to confirm this statement.

\section{Acknowledgements}

The present study was supported in part by the Spanish Ministerio de Economía y Competitividad (AGL2010-22319-C03, AGL2013-49083-C3-1-R) and by the Generalitat de Catalunya (2014 SGR 773). The Ministerio de Economía y Competitividad, (CIBEROBN) Instituto de Salud Carlos III and the Generalitat de Catalunya had no role in the design, analysis or writing of this article.

Contributions of authors were as follows: All authors had full access to all the data in the study and take responsibility for the integrity of the data and the accuracy of analyses. All authors were involved in the conception and design of the study, the collection and assembly of data and the analysis and interpretation of the data. N. O.-L. and E. E.-F. wrote the article but the intellectual content of the manuscript was critically revised by all the authors.

None of the authors has any conflicts of interest. 


\section{References}

1. Xu H, Kulkarni KH, Singh R, et al. (2009) Disposition of naringenin via glucuronidation pathway is affected by compensating efflux transporters of hydrophilic glucuronides. Mol Pharm 6, 1703-1715.

2. Yoshimura M, Sano A, Kamei J, et al. (2009) Identification and quantification of metabolites of orally administered naringenin chalcone in rats. J Agric Food Chem 57, 6432-6437.

3. Felgines C, Texier O, Morand C, et al. (2000) Bioavailability of the flavanone naringenin and its glycosides in rats. $A m J$ Physiol Gastrointest Liver Physiol 279, G1148-G1154.

4. Chabane MN, Al Ahmad A, Peluso J, et al. (2009) Quercetin and naringenin transport across human intestinal Caco-2 cells. J Pharm Pharmacol 61, 1473-1483.

5. Amidon GL, Lennernäs H, Shah VP, et al. (1995) A theoretical basis for a biopharmaceutic drug classification: the correlation of in vitro drug product dissolution and in vivo bioavailability. Pharm Res 12, 413-420.

6. Azuma K, Ippoushi K, Ito H, et al. (2002) Combination of lipids and emulsifiers enhances the absorption of orally administered quercetin in rats. J Agric Food Chem 50, 1706-1712.

7. Jeong EJ, Jia X \& Hu M (2005) Disposition of formononetin via enteric recycling: metabolism and excretion in mouse intestinal perfusion and Caco-2 cell models. Mol Pharm 2, 319-328.

8. Escribano E, García Sala X, Salamanca J, et al. (2012) Singlepass intestinal perfusion to establish the intestinal permeability of model drugs in mouse. Int J Pharm 436, 472-477.

9. Lieber CS, Leo MA, Mak KM, et al. (2004) Model of nonalcoholic steatohepatitis. Am J Clin Nutr 79, 502-509.

10. Vallverdú-Queralt A, De Alvarenga JF, Estruch R, et al. (2013) Bioactive compounds present in the Mediterranean sofrito. Food Chem 41, 3365-3372.

11. Martínez-Huélamo M, Tulipani S \& Torrado X (2012) Validation of a new LC-MS/MS method for the detection and quantification of phenolic metabolites from tomato sauce in biological samples. I Agric Food Chem 60, 4542-4549.

12. Dahan A, West BT \& Amidon GL (2009) Segmental-dependent membrane permeability along the intestine following oral drug administration: evaluation of a triple single-pass intestinal perfusion (TSPIP) approach in the rat. Eur $J$ Pharm Sci 36, 320-329.

13. Eisen EJ (1976) Results of growth curve analyses in mice and rats. J Anim Sci 42, 1008-1023.

14. Miglioli PA, Allerberger F, Calabró GB, et al. (2001) Effects of daily oral administration of rifaximin and neomycin on faecal aerobic flora in rats. Pharmacol Res 44, 373-375.

15. Scarpignato C \& Pelosini I (2005) Rifaximin, a poorly absorbed antibiotic: pharmacology and clinical potential. Chemotherapy 51, Suppl. 1, S36-S66.

16. Tulipani S, Martinez-Huelamo M, Rotches Ribalta M, et al. (2012) Oil matrix effects on plasma exposure and urinary excretion of phenolic compounds from tomato sauces: evidence from a human pilot study. Food Chem 130, 581-590.

17. Varma MVS \& Panchagnula R (2005) Prediction of in vivo intestinal absorption enhancement on $P$-glycoprotein inhibition, from rat in situ permeability. J Pharm Sci 94, $1694-1704$

18. Zakeri-Milani $\mathrm{P}$, Valizadeh $\mathrm{H}$, Tajerzadeh $\mathrm{H}$, et al. (2007) Predicting human intestinal permeability using single-pass intestinal perfusion in rat. J Pharm Sci 10, 368-379.
19. Sutton SC, Rinaldi MTS \& Vukovinsky KE (2001) Comparison of the gravimetric, phenol red, and 14C-PEG-3350 methods to determine water absorption in the rat single-pass intestinal perfusion model. AAPS Pharm Sci 3, 1-5.

20. Japar D, Wu SP, Hu Y, et al. (2010) Significance and regional dependency of peptide transporter (PEPT) 1 in the intestinal permeability of glycylsarcosine: in situ single-pass perfusion studies in wild-type and Pept1 knockout mice. Drug Metab and Dispos 38, 1740-1746.

21. Incecayir T, Tsume Y \& Amidon GL (2013) Comparison of the permeability of metoprolol and labetalol in rat, mouse, and Caco-2 cells: use as a reference standard for BCS classification. Mol Pharm 10, 958-966.

22. Masaoka Y, Tanaka Y, Kataoba M, et al. (2006) Site of absorption after oral administration: assessment of membrane permeability and luminal concentration of drugs in each segment of gastrointestinal tract. Eur J Pharm Sci 29, 240-250.

23. McConnell EL, Basit AW \& Murdan S (2008) Measurements of rat and mouse gastrointestinal $\mathrm{pH}$, fluid and lymphoid tissue, and implications for in vivo experiments. J Pharm Pharm 60, 63-70

24. Komiya I, Park JY, Kamani A, et al. (1980) Quantitative mechanistic studies in simultaneous fluid flow and intestinal absorption using steroids as model solutes. Int J Pharm $\mathbf{4}$, 249-262.

25. Vallverdú-Queralt A, Regueiro J, Martinez-Huelamo, et al. (2014) A comprehensive study on the phenolic profile of widely used culinary herbs and spices: rosemary, thyme, oregano, cinnamon, cumin and bay. Food Chem 154, 299-307.

26. DrugBank (2014) DrugBank database (open data drug and drug target data base). http://www.drugbank.ca/drugs/ DB03467 (accessed May 2014).

27. Fagerholm U, Johansson M \& Lennernäs H (1996) Comparison between permeability coefficients in rat and human jejunum. Pharm Res 13, 1336-1342.

28. Takanaga H, Ohnishi A, Matsuo H, et al. (1998) Inhibition of vinblastine efflux mediated by $P$-glycoprotein by grapefruit juice components in Caco-2 cells. Biol Pharm Bull 21, 1062-1066.

29. Bailey DG, Malcolm J, Arnold O, et al. (1998) Grapefruit juice-drug interactions. Br J Clin Pharmacol 46, 101-110.

30. Le Goff N, Koffel JC, Vandenschrieck S, et al. (2002) Comparison of in vitro hepatic models for the prediction of metabolic interaction between simvastatin and naringenin. Eur J Drug Metab Pharmacokinet 27, 233-241.

31. Le Goff-Klein N, Koffel JC, Jung L, et al. (2005) In vitro inhibition of simvastatin metabolism, a HMG-CoA reductase inhibitor in human and rat liver by bergamottin, a component of grapefruit juice. Eur J Pharm Sci 18, 31-35.

32. Hsiu SL, Huang TY, Hou YC, et al. (2002) Comparison of metabolic pharmacokinetics of naringin and naringenin in rabbits. Life Sci 70, 1481-1489.

33. Chen G, Zhang D, Jing N, et al. (2003) Human gastrointestinal sulfotransferases: identification and distribution. Toxicol Appl Pharmacol 187, 186-197.

34. Gregory P, Lewinsky R, Gardner-Stephen D, et al. (2004) Regulation of UDP glucuronosyltransferases in the gastrointestinal tract. Toxicol Appl Pharmacol 199, 354-356.

35. Fang T, Wang Y, Ma Y, et al. (2006) A rapid LC/MS/MS quantitation assay for naringin and its two metabolites in rats plasma. J Pharm Biomed Anal 40, 454-459.

36. Manach C, Scalbert A, Morand C, et al. (2004) Polyphenols: food sources and bioavailability. Am J Clin Nutr 79, $727-747$. 
37. Rechner AR, Smith MA, Kuhnle G, et al. (2004) Colonic metabolism of dietary polyphenols: influence of structure on microbial fermentation products. Free Radic Biol Med 36, 212-225.

38. Possimiers S, Bolca S, Verstraete W, et al. (2011) The intestinal microbiome: a separate organ inside the body with the metabolic potential to influence the bioactivity of botanicals. Fitoterapia 82, 53-66.

39. Boto-Ordóñez M, Rothwell JA, Andres-Lacueva C, et al. (2014) Prediction of the wine polyphenol metabolic space: an application of the Phenol-Explorer database. Mol Nutr Food Res 58, 466-477.

40. Cong D, Fong AK, Lee R, et al. (2001) Absorption of benzoic acid in segmental regions of the vascularly perfused rat small intestine preparation. Drug Metab Dispos 29, 1539-1547.

41. Gao K, Xu A, Krul C, et al. (2006) Of the major phenolic acids formed during human microbial fermentation of tea, citrus, and soy flavonoid supplements, only 3, 4-dihydroxyphenylacetic acid has antiproliferative activity. J Nutr 136, 52-57.

42. Aura AM (2008) Microbial metabolism of dietary phenolic compounds in the colon. Phytochem Rev 7, 407-429.
43. Serra A, Macià A, Romero MP, et al. (2012) Metabolic pathway of the colonic metabolism of flavonoids (flavonols, flavones and flavonones) and phenolic acids. Food Chem 130, 383-393.

44. Konishi Y \& Kobayashi S (2004) Microbial metabolites of ingested caffeic acid are absorbed by the monocarboxylic acid transporter (MCT) in intestinal Caco-2 cell monolayers. J Agric Food Chem 52, 6418-6424.

45. Spoelstra SF (1978) Degradation of tyrosine in anaerobically stored piggery wastes and in pig feces. Appl Environ Microbiol 36, 631-638.

46. Lord RS \& Bradley JA (2008) Clinical applications of urinary organic acids. Part 2. Dysbiosis markers. Altern Med Rev 13, 292-306.

47. Gao J, Gillilland MIII \& Owyang C (2014) Rifaximin, gut microbes and mucosal inflammation: unravelling a complex relationship. Gut Microbes 5, 571-575.

48. Miene C, Weise A \& Glei M (2011) Impact of polyphenol metabolites produced by colonic microbiota on expression of COX-2 and GSTT2 in human colon cells (LT97). Nutr Cancer 63, 653-662. 